\title{
Éditorial
}

\section{La sélection en Master}

Tempête sur l'enseignement supérieur. L'ensemble des Conférences de Doyens a récemment affirmé que la qualité de la formation au niveau master nécessitait, à quelque niveau que ce soit, un processus de sélection des étudiants. Jusqu'à présent cette sélection s'opérait généralement au niveau de l'entrée en seconde année. Il a été objecté à cette pratique que depuis la mise en place du LMD le master constituait un cycle d'enseignement et qu'une sélection à miparcours n'était pas concevable. Les Conférences de Doyens ont alors proposé d'envisager, du moins pour les diplômes subissant une forte pression, le principe d'une sélection à l'entrée du Master.

Les grades de Licence et de Master attestent de compétences nettement différenciées. Par exemple dans l'offre de formation STAPS, La licence forme essentiellement des professionnels destinés à intervenir directement auprès du public. Le master vise à former des personnels d'encadrement et des chefs de projets. Le grade de Master suppose l'accession à un niveau de compétence attesté notamment par la réalisation de stages en responsabilité et la poursuite d'un travail encadré d'étude et de recherche débouchant sur la production d'un mémoire. Le maintien de la qualité de la formation demande un taux d'encadrement satisfaisant pour les travaux de recherche et un nombre suffisant de lieux de stage. Il semble en effet difficile d'atteindre cet objectif sans qu'un processus de sélection soit mis en place, à un endroit ou un autre du cursus. Il semble en effet que le seuil logique de sélection se situe à l'entrée de la première année de Master. Une telle proposition mérite évidemment d'être discutée en profondeur, et notamment en ce qui concerne les critères pouvant présider à cette sélection précoce (résultats de Licence, projet professionnel, etc.). Cette sélection ne peut être assimilée de toutes évidences aux processus mis en œuvre dans les formations à capacité d'accueil limitée (tirage au sort). Elle devrait être associée à une démarche d'orientation active, associant de manière individualisée les étudiants.

Les associations étudiantes proposent, pour leur part, la suppression de toute sélection, l'entrée et la poursuite des études en Master étant pensées comme un droit pour tout titulaire de la Licence, tout comme l'entrée à l'université est un droit pour tout titulaire du baccalauréat.

Il y a là une opposition frontale de conceptions vis-à-vis des missions de l'enseignement supérieur. Les universitaires se voient taxés d'élitisme, quand ils ne font guère que tenter avec les moyens limités qu'on leur alloue de maintenir la crédibilité de leurs formations, en terme d'adossement à la recherche et d'insertion professionnelle. D'un autre côté, les associations étudiantes militent logiquement, mais avec une dose manifeste d'utopie, pour l'accès libre aux formations supérieures.

Le ministère semble à l'heure actuelle ignorer les réserves des universités et refuser toute idée de sélection en master. On peut d'étonner de cet autisme vis-à-vis des arguments de ceux qui in fine font fonctionner le système. Les universitaires font preuve d'une adaptabilité remarquable pour faire face à la massification de l'enseignement supérieur. Ils ont su tourner le dos aux traditions de l'enseignement académique pour rentrer dans des perspectives plus proches des besoins des usagers, en termes de professionnalisation et d'ouverture aux réalités socio-économiques. Ils s'attachent à prendre en compte la diversité des publics qui peuplent les amphithéâtres, afin de permettre la réussite du plus grand nombre. Tout cela dans un contexte de restrictions budgétaires qui bloque rapidement tout initiative trop dispendieuse.

Il peut être tentant d'acheter la paix sociale sur le dos des universitaires. Mais ces derniers finiront peut être par se lasser de devoir en permanence satisfaire des exigences contradictoires. Et la qualité des formations n'y gagnera pas grand chose. 